\title{
Intrinsic and extrinsic contributions to magnetodielectric effect in double perovskite $\mathrm{La}_{2} \mathrm{CoMnO}_{6}$ nanoparticles
}

\author{
A. Venimadhav $\cdot$ Devi Chandrasekar · \\ J. Krishna Murthy
}

Received: 1 November 2011/Accepted: 14 February 2012/Published online: 6 March 2012

(C) The Author(s) 2012. This article is published with open access at Springerlink.com

\begin{abstract}
We have studied the magnetic and magnetodielectric (MD) properties of $\mathrm{La}_{2} \mathrm{CoMnO}_{6}$ nanoparticles of size $\sim 28 \mathrm{~nm}$ prepared by sol-gel method. Dielectric behavior showed two relaxations coinciding with the magnetic transitions at 218 and $150 \mathrm{~K}$, respectively. At those relaxation maxima, a large MD effect of 11 and $8 \%$ (at $100 \mathrm{kHz}$ ) with $5 \mathrm{~T}$ was observed and the magnitude of MD effect is dependent on frequency. $\Delta \varepsilon_{\mathrm{MD}} \propto M^{2}$ relation in the paramagnetic to ferromagnetic regime suggests the spin lattice coupling; however, within the ferromagnetic region $(200-100 \mathrm{~K})$, we have found $\Delta \varepsilon_{\mathrm{MD}} \propto M^{4}$ relation which may be due to the complicated spin-pair correlation interactions among next nearest neighbors. Complex impedance analysis showed large frequency dispersion of dielectric permittivity, and strong influence of both bulk and grain boundary impedances with magnetic field clearly suggests that both intrinsic spin-lattice coupling and extrinsic Maxwell-Wagner model combined with magnetoresistance effect contribute to the observed MD property.
\end{abstract}

Keywords Magnetodielectric effect .

$\mathrm{La}_{2} \mathrm{CoMnO}_{6}$ nanoparticles $\cdot$ Spin-lattice coupling .

Maxwell-Wagner model

A. Venimadhav · D. Chandrasekar · J. Krishna Murthy ( $\square)$ Cryogenic Engineering Centre, IIT Kharagpur,

Kharagpur 721302, India

e-mail: 09cr9701@iitkgp.ac.in

D. Chandrasekar

Department of Physics, IIT Kharagpur,

Kharagpur 721302, India

\section{Introduction}

Materials that posses change in dielectric properties with an applied external magnetic field, namely magnetodielectric (MD) effect, are potential in several novel functional device applications (Eerenstein et al. 2006). MD effect has been observed in systems where the electrical dipoles get coupled to magnetic order with or without long range electrical dipolar ordering (both multiferroics $\mathrm{BiFe}$ $\mathrm{O}_{3}, \mathrm{TbMnO}_{3}$ and non multiferroics $\mathrm{SeCuO}_{3}, \mathrm{La}_{2} \mathrm{NiMnO}_{6}$ ) (Goswami et al. 2011; Kagawa et al. 2011; Lawes et al. 2009, Rogado et al. 2005). Though, MD effect has been studied in wide varieties of systems, the origin of this effect can be different depending on the electronic structure and phonon spectrum of the system. The reported large MD effect near the FM ordering $(\sim 100 \mathrm{~K})$ in $\mathrm{BiMnO}_{3}$ system and the observed MD is proportional to the square of the magnetic-order parameter, (i.e., $\Delta \varepsilon_{\mathrm{MD}} \sim \gamma M^{2}$ ) suggesting the intrinsic MD effect due to the magnetoelectric coupling (Kimura et al. 2003). However, extrinsic effects like particle size, grain boundaries and spin disorder etc., can also influence the dielectric response under magnetic field. Recently, the extrinsic contribution of Maxwell-Wagner (MW) interfacial effect combined with the negative magnetoresistance (MR) can give rise to a large MD effect, even though the material may not be multiferroic (Catalan 2006).

The family of double perovskite systems with formula $\mathrm{A}_{2} \mathrm{BB}^{\prime} \mathrm{O}_{6}\left(\mathrm{~A}-\mathrm{Sr}, \mathrm{Bi}, \mathrm{La}\right.$; $\mathrm{B} / \mathrm{B}^{\prime}-\mathrm{Ni}, \mathrm{Fe}, \mathrm{Mn}, \mathrm{Co}, \mathrm{Mo}$ ) has received renewed interest due to their correlation of magnetic and dielectric properties (Rogado et al. 2005; Kobayashi et al. 1999; Dass and Goodenough 2003). The $\mathrm{La}_{2} \mathrm{CoMnO}_{6}$ (LCMO) is such a system which exhibits FM transition around $\sim 220 \mathrm{~K}$ (Dass and Goodenough 2003) and their epitaxial thin films showed a MD effect around its

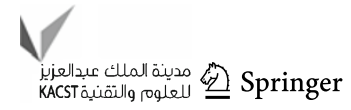


FM transition, and the effect was attributed to the spinlattice coupling (Singh M et al. 2007). Truong et al. (2007) have observed softening of phonon modes near the magnetic ordering temperatures in LCMO system confirming the existence of spin-lattice coupling. Here in this report, we show the preparation of LCMO nanoparticles and their MD effect near the magnetic transitions, and also possible reasons of intrinsic and extrinsic contributions to MD effect are discussed.

\section{Experimental details}

The polycrystalline single phase LCMO samples with $28 \mathrm{~nm}$ sizes were prepared by conventional sol gel method. The required stoichiometry ratio of $\mathrm{La}_{2} \mathrm{O}_{3}$, cobalt nitrate $\left(\mathrm{Co}\left(\mathrm{NO}_{3}\right)_{2} \cdot 6 \mathrm{H}_{2} \mathrm{O}\right)$ and manganese acetate $\left(\left(\mathrm{CH}_{3} \mathrm{COO}\right)_{2}\right.$ $\mathrm{Mn} \cdot 4 \mathrm{H}_{2} \mathrm{O}$ ) was dissolved in water and added appropriate amount of ethylene glycol which acts as a chelating agent, the resultant mixture was kept at $80^{\circ} \mathrm{C}$ with continuous stirring for $2 \mathrm{~h}$. The obtained viscous gel was decomposed at $150^{\circ} \mathrm{C}$ temperature for $6 \mathrm{~h}$. The finally obtained precursor was calcinated at $600^{\circ} \mathrm{C}$ for $2 \mathrm{~h}$ to obtain LCMO nanoparticles. The temperature dependence of $\mathrm{AC}$ susceptibility was measured with Quantum design SQUIDVSM magnetometer. The temperature and the field variation of dielectric properties were measured using HIOKI-LCR meter at various frequencies $(100 \mathrm{~Hz}-100 \mathrm{kHz})$ with an excitation AC voltage of $0.5 \mathrm{~V}$ using a closed cycle cryofree superconducting system from 300 to $5 \mathrm{~K}$.

\section{Results and discussion}

The structural analysis (not shown) by XRD and TEM has revealed that the prepared LCMO nanoparticles are of the order of $\sim 28 \mathrm{~nm}$ and showed single phase with no impurities; the structure can be assigned to pseudo tetragonal crystal structure (Dass and Goodenough 2003). Detailed structural analysis and surface morphological studies are reported elsewhere (Krishna murthy and Venimadhav 2012). Figure 1a shows the temperature dependent out-of-phase $\left(\chi^{\prime \prime}\right)$ component of AC susceptibility for $523 \mathrm{~Hz}$ with amplitude of AC signal 1 Oe. Here $\chi^{\prime \prime}$ component is sharp and abruptly decreases to minimum at $218 \mathrm{~K}$; and this can be assigned as first ferromagnetic transition $\left(T_{\mathrm{c} 1}\right)$ and the second magnetic transition at $\left(T_{\mathrm{c} 2}\right)$ $\sim 135 \mathrm{~K}$ is clear but weak, and this signal is difficult to distinguish against the large signal from the $218 \mathrm{~K}$ transition. In addition to the above two ferromagnetic transitions, there is one more very low temperature magnetic glassy transition and the details of which is being currently investigated. The two magnetic transitions at 135 and

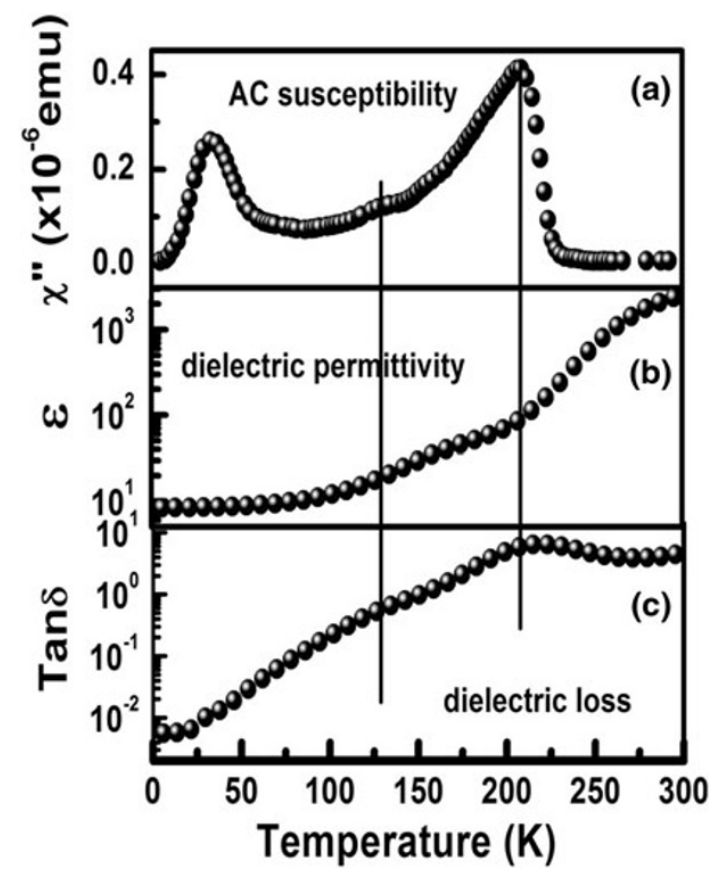

Fig. $1 a-c$ shows temperature dependence of out of phase component of AC susceptibility, dielectric permittivity and dielectric loss, respectively

$218 \mathrm{~K}$ are in agreement with the previous reports of bulk and films (Dass and Goodenough 2003; Singh et al. 2007), and are assigned to ordering of $\mathrm{Co}^{3+}-\mathrm{O}-\mathrm{Mn}^{3+}$ and $\mathrm{Co}^{2+}$ $\mathrm{O}-\mathrm{Mn}^{4+}$ valance states, respectively. Temperature dependent real part of dielectric permittivity $(\varepsilon)$ for $100 \mathrm{kHz}$ frequency is shown in Fig. 1b. The dielectric permittivity has been found to be large at room temperature in comparison to LCMO films (Singh et al. 2007). It is interesting to note that there are two sharp drops in the dielectric permittivity accompanied by clear relaxation peaks in the dielectric loss as shown in the Fig. 1c near the magnetic transitions. The dielectric permittivity saturates $(\varepsilon \sim 10)$ at very low temperature below $20 \mathrm{~K}$ due to dipolar freezing.

MD behavior as a function of applied magnetic field at different temperatures (isothermal plots) for $100 \mathrm{kHz}$ frequency is shown in the Fig. 2a. The MD\% effect has been calculated as $\operatorname{MD}(\%)=\left[\frac{\varepsilon_{H(T)}-\varepsilon_{0(T)}}{\varepsilon_{0(T)}}\right] \times 100$. As shown in this figure, the observed MD effect increases with increasing of field and it shows two maximum one with $\sim 8 \%$ at the weak FM transition $\sim 135 \mathrm{~K}$ and second one $\sim 10 \%$ occurs around the predominate $\mathrm{FM}$ transition at $220 \mathrm{~K}$ under $5 \mathrm{~T}$ field. Field dependence of magnetodielectric loss (i.e., $\operatorname{MDL}(\%)=\left[\frac{\operatorname{Tan} \delta_{H(T)}-\operatorname{Tan} \delta_{0(T)}}{\operatorname{Tan} \delta_{0(T)}}\right] * 100$ ) at $100 \mathrm{kHz}$ for different temperatures is shown in the Fig. $2 \mathrm{~b}$. It is interesting to note that both MD effect and MDL are increasing with field for all temperatures below the FM transition at $\sim 218 \mathrm{~K}$, but they showing different sign 


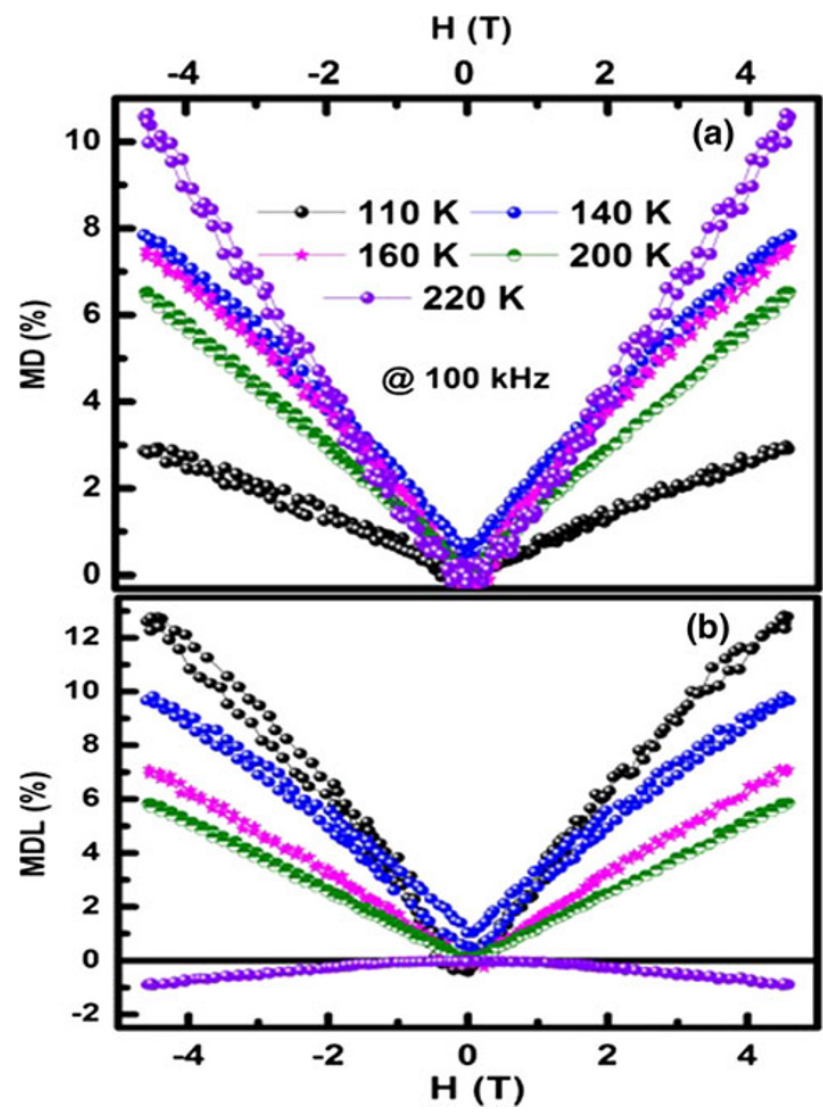

Fig. $2 a, b$ shows magnetic field dependence of MD effect and MDL at $100 \mathrm{kHz}$ frequency for different temperatures

above the FM transition ( $\geq 220 \mathrm{~K})$. This suggests that the observed MD effect in LCMO nanoparticles may be an intrinsic effect.

In order to understand the origin of magnetic fieldinduced dielectric constant in these LCMO nanoparticles, we can incorporate Ginzburg-Landau theory for second order phase transition analysis. In case of ferroelectromagnets, the thermodynamic potential can be as follows,

$\begin{aligned} \Phi= & \varphi_{0}+\alpha P^{2}+\frac{\beta}{2} P^{4}-P E+\alpha^{\prime} M^{2}+\frac{\beta^{\prime}}{2} M^{4}-M H \\ & +\gamma P^{2} M^{2}\end{aligned}$

where $P, M, E$ and $H$ are the polarization, magnetization, electric field and magnetic field, respectively and $\alpha, \beta, \alpha^{\prime}$, $\beta^{\prime}, \gamma$ are the coefficients of Landau free energy terms and their nature can be dependent on type of magnetic phase transition. According to Ginzburg-Landau phenomenological Eq. (1), $\varepsilon_{\mathrm{MD}}$ is proportional to spin-pair correlation function and $M^{2}$ with a relation, $\mathrm{MD}(\%)=\gamma M^{2}$. Figure $3 \mathrm{a}$ shows a linear relation between $\mathrm{MD}(\%)$ and the square of magnetization $\left(M^{2}\right)$ near the PM to FM transition region $\sim 220 \mathrm{~K}$ for $100 \mathrm{kHz}$. However, in the present system, this relation does not hold below the FM transition due to complicated spin-pair correlation interactions among next

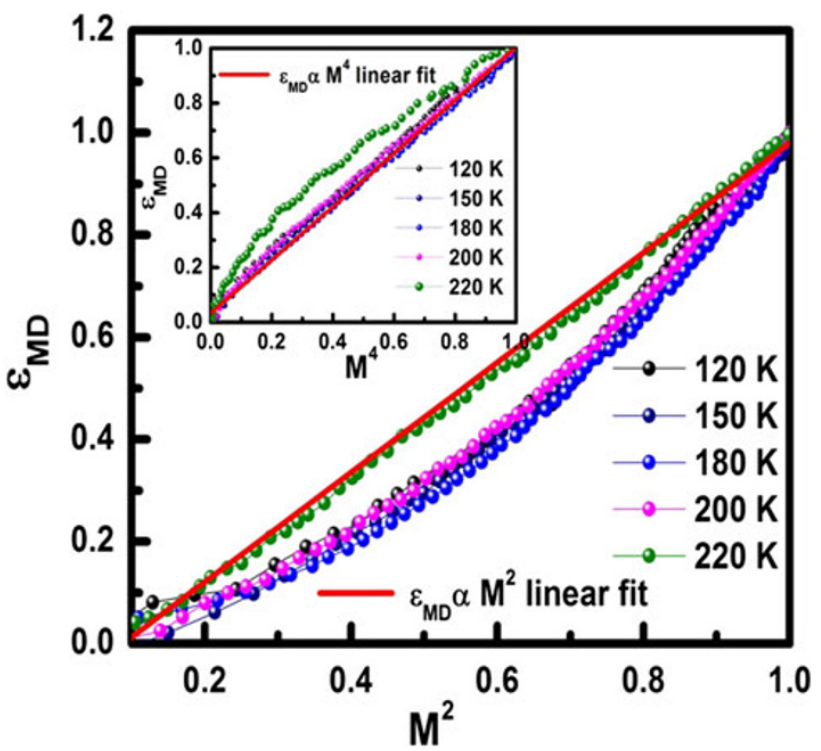

Fig. 3 shows variation of MD effect with $M^{2}$ and $M^{4}$ (inset) relation for $100 \mathrm{kHz}$ frequency at different temperatures near to FM-PM transition and below the FM transition temperatures, respectively

nearest neighbours. As shown in inset to Fig. 3a, it can be observed that a higher order magnetization of $M^{4}$ fits well with the MD (\%) in the FM region from 200 to $100 \mathrm{~K}$ for $100 \mathrm{kHz}$. Recently, such a higher order coupling of $M^{4}$ with MD (\%) was observed in thin film multilayers (Padhan et al. 2009) and composites of PCMO nanoparticles with polymers (Chandrasekhar et al. 2011). Moreover, such a higher order effect is possible in low dimensional materials (Eerenstein et al. 2006). Further theoretical and experimental study would be required to understanding clearly the origin behind such a higher order coupling of MD effect in FM regime.

We have measured frequency dependence of complex quantity of impedance $(Z)$ with a function of frequency at different temperatures. Figure 4 shows (i.e., Nyquist plots) the variation of real $\left(Z^{\prime}\right)$ and imaginary parts $\left(Z^{\prime \prime}\right)$ of impedance at different temperatures with (5T) and without field (0T). For every temperature, we have observed two semi arc circles correspond to bulk contribution to dielectric permittivity in the high frequency range and grain boundary (GB), i.e., interfacial region contribution to the dielectric permittivity in the low frequency range. But at low temperature around $120 \mathrm{~K}$, the bulk contribution semi arc is predominant, while by increasing the temperature its effect decreases and contribution from GB semi arc is dominant. Under the application of magnetic field, both core dominated bulk impedance and interface dominated GB impedance changes. The shift of impedance arcs corresponds to bulk and GB regions under the magnetic field demonstrates that the MD effect is related to both intrinsic and extrinsic effects (Dong et al. 2011). And the shift of the 


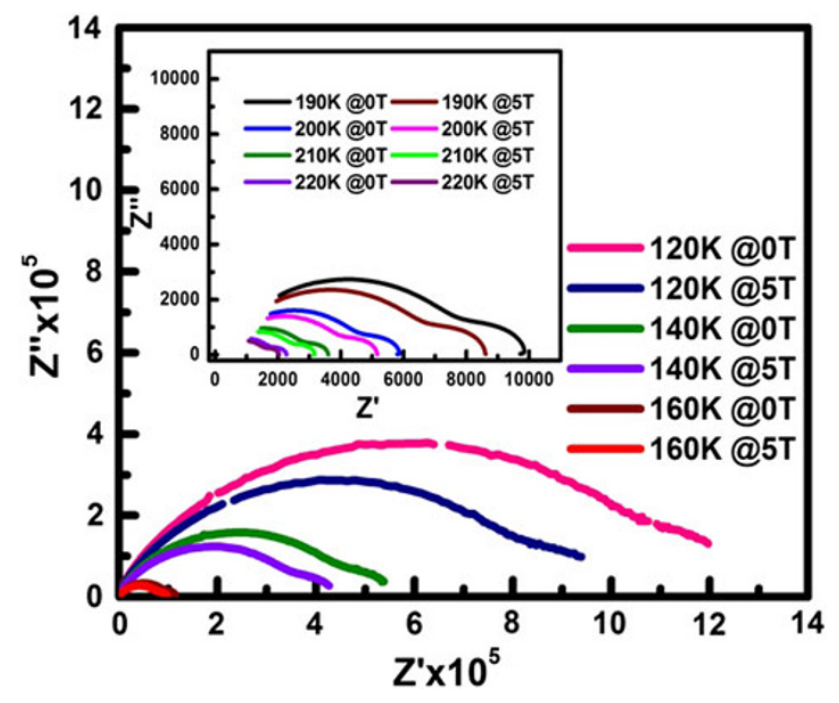

Fig. 4 Variation of real and imaginary parts of impedance (i.e., complex impedance plane) with and without applied magnetic field of $5 \mathrm{~T}$ for different temperatures

bulk arc can be attributed to the intrinsic spin lattice coupling. In the present LCMO polycrystalline nanoparticle system, the growth of magnetic domains in the applied magnetic fields giving rise to decrement of probability of domain wall scattering and the spin disorder at the GB regions, and this strongly influence the resistance which leads to negative MR property. So, the change in the GB resistance as a function of temperature and field suggests that the conduction mechanism can play a role in the observed MD effect.

In summary, we have studied the MD behavior of LCMO nanoparticles in the PM and FM regime. A large MD response of 11 and $8 \%$ at $100 \mathrm{kHz}$ near the two FM transitions was observed. The MD response was alleged to arise due to the dipolar relaxation coupling with magnetic order parameter. Though MD effect shows $\Delta \varepsilon_{\mathrm{MD}} \propto M^{2}$ in the PM to FM transition regime, $\Delta \varepsilon_{\mathrm{MD}} \propto M^{4}$ was observed in the FM region up to $100 \mathrm{~K}$. Strong influence of GB and bulk impedance with magnetic field suggests that both intrinsic coupling of magnetic with dielectric orders and extrinsic effect from MW model combined with MR property will contributes to the observed MD effect in the LCMO nanoparticles.

Acknowledgments This work was supported by DST (SR/FTP/PS36/2007) and the authors also acknowledge IIT Kharagpur for funding
VSM SQUID magnetometer and DST, New Delhi for FIST grant for establishing cryogen free high magnetic field facility. Krishnamurthy thanks CSIR-UGC, Delhi for JRF.

Open Access This article is distributed under the terms of the Creative Commons Attribution License which permits any use, distribution, and reproduction in any medium, provided the original author(s) and the source are credited.

\section{References}

Catalan G (2006) Magnetocapacitance without magnetoelectric coupling. Appl Phys Lett 88(1-3):102902

Chandrasekhar KD, Das AK, Venimadhav A (2011) Large magnetodielectric response in $\operatorname{Pr}_{0.6} \mathrm{Ca}_{0.4} \mathrm{MnO}_{3}$ /polyvinyle denefluoride nanocomposites. Appl Phys Lett 98(1-3):122908

Dass RI, Goodenough JB (2003) Multiple magnetic phases of $\mathrm{La}^{2} \mathrm{CoMnO}_{6-\delta}$. Phys Rev B 67(1-9):014401

Dong S, Hou Y, Yao Y, Yin Y, Ding D, Yu Q, Li X (2011) Magnetodielectric effect and tunable dielectric properties LaMn1-xFexO3. J Am Ceram Soc 93(11):3418-3814

Eerenstein W, Mathura ND, Scott JF (2006) Multiferroic and magnetoelectric materials. Nature 442:759-765

Goswami S, Bhattacharya D, Choudhury P, Ouladdiaf B, Chatterji T (2011) Multiferroic coupling in nanoscale $\mathrm{BiFeO}_{3}$. Appl Phy Lett 99(1-3):073106

Kagawa F, Onose Y, Kaneko Y, Tokura Y (2011) Relaxation dynamic of multiferroic domain walls in DyMnO3 with cycloidal spin order. Phy Rev B 83(1-10):054413

Kimura T, Kawamoto S, Yamada I, Azuma M, Takano M, Tokura Y (2003) Magnetocapacitance effect in multiferroic in $\mathrm{BiMnO}_{3}$. Phy Rev B 67(1-4):180401(R)

Kobayashi KI, Kimura T, Tomioka Y, Saeada H, Terakura K (1999) Intergrain tunneling magnetoresistance in polycrystals of the ordered perovskite $\mathrm{Sr}_{2} \mathrm{FeMoO}_{6}$. Phy Rev B 59:1159-11162

Krishna murthy J, Venimadhav A (2012) Magnetodielectric behaviour in La2CoMnO6 nanoparticles. J Appl Phys 111(1-5):024102

Lawes G, Kimura T, Varma CM, Subramanian MA, Rogado N, Cava RJ, Ramirez AP (2009) Magnetodielectrics effect at the magnetic ordering transitions. Prog Solid State Chem 37:40-54

Padhan P, LeClair P, Gupta A, Subramanian MA, Srinivasan G (2009) Magnetodielectric effect in $\mathrm{Bi}_{2} \mathrm{NiMnO}_{6}-\mathrm{La}_{2} \mathrm{NiMnO}_{6}$ superlattices. J Condens Matter 21(1-7):306004

Rogado NS, Li J, Sleight AW, Subramanian MA (2005) Magnetocapacitance and magnetoresistance near to room temperature in ferromagnet semiconductor: La2NiMnO6. Adv Mater 17:2225-2227

Singh MP, Truong KD, Fournier P (2007) Magnetodielectric effect in double perovskite $\mathrm{La}_{2} \mathrm{CoMnO}_{6}$ thin films. Appl Phy Lett 91(1-3):042504

Truong KD, Laverdière J, Singh MP, Jandl S, Fournier P (2007) Impact of $\mathrm{Co} / \mathrm{Mn}$ cation ordering on phonon anomalies in $\mathrm{La}_{2} \mathrm{CoMnO}_{6}$ double perovskites: Raman spectroscopy. Phys Rev B 76(1-4):132413 